\title{
Multiparametric Molecular Imaging Provides Mechanistic Insights into Sympathetic Innervation Impairment in the Viable Infarct Border Zone
}

\author{
Riikka Lautamaki*1, Tetsuo Sasano*2, Takahiro Higuchi ${ }^{1}$, Stephan G. Nekolla ${ }^{3}$, Albert C. Lardo $^{2}$, Daniel P. Holt ${ }^{1}$, \\ Robert F. Dannals ${ }^{1}$, M. Roselle Abraham ${ }^{2}$, and Frank M. Bengel ${ }^{1,4}$ \\ ${ }^{1}$ Division of Nuclear Medicine, Russell H. Morgan Department of Radiology, Johns Hopkins University, Baltimore, Maryland; \\ ${ }^{2}$ Division of Cardiology, Department of Medicine, Johns Hopkins University, Baltimore, Maryland; ${ }^{3}$ Nuklearmedizinische Klinik, \\ Technische University of Munich, Munich, Germany; and ${ }^{4}$ Department of Nuclear Medicine, Hannover Medical School, Hannover, Germany
}

\begin{abstract}
Impaired catecholamine handling in the viable infarct border zone may play an important role in ventricular remodeling and lethal arrhythmia. We sought to get further biologic insights into cardiac sympathetic neuronal pathology after myocardial infarction, using multiple tomographic imaging techniques. Methods: In a porcine model of myocardial infarction $(n=13)$, PET and MR imaging were performed after 4-6 wk and integrated with electrophysiologic testing and postmortem histology. Results: PET with the physiologic neurotransmitter ${ }^{11} \mathrm{C}$-epinephrine, which is sensitive to metabolic degradation unless it is stored and protected in neuronal vesicles, identified a defect exceeding the perfusion defect (defined by ${ }^{13} \mathrm{~N}-$ ammonia; defect size in all animals, $42 \pm 12$ vs. $35 \% \pm 12 \%$ of left ventricle, $P<0.001$ ). In a subgroup of 7 animals, defect of the metabolically resistant catecholamine ${ }^{11} \mathrm{C}$-hydroxyephedrine was smaller than epinephrine $(41 \pm 8$ vs. $47 \% \pm 6 \%$ of left ventricle, $P=$ 0.004 ), whereas defect of a third catecholamine, ${ }^{11} \mathrm{C}$-phenylephrine, which is sensitive to metabolic degradation, was similar to epinephrine ( $48 \pm 6$ vs. $47 \% \pm 6 \%, P=0.011$ vs. perfusion defect). Histology confirmed the presence of nerve fibers in the infarct border zone. Tagged MR imaging identified impaired peak circumferential wall strain and wall thickening in myocardial segments with epinephrine/perfusion mismatch $(n=6)$. Confirmatory of prior work, inducible ventricular tachycardia was associated with a larger epinephrine/perfusion mismatch $(n=11)$. Conclusion: In the viable infarct border zone, neuronal vesicular catecholamine storage and protection from metabolic degradation are more severely altered than catecholamine uptake. This alteration may reflect an intermediate state between normal innervation and complete denervation in advanced disease.
\end{abstract}

Key Words: positron emission tomography; myocardial infarction; sympathetic innervation; magnetic resonance imaging

J Nucl Med 2015; 56:457-463

DOI: 10.2967/jnumed.114.149971

Received Oct. 14, 2014; revision accepted Jan. 5, 2015.

For correspondence or reprints contact: Frank M. Bengel, Department of Nuclear Medicine, Hannover Medical School, Carl-Neuberg-Strasse 1, D-30625 Hannover, Germany.

E-mail: bengel.frank@mh-hannover.de

${ }^{*}$ Contributed equally to this work.

Published online Jan. 29, 2015.

COPYRIGHT (c) 2015 by the Society of Nuclear Medicine and Molecular Imaging, Inc.
$\mathbf{P}$ revious studies have suggested that myocardial sympathetic innervation is compromised after myocardial infarction (1-3). High sensitivity of nerve terminals to ischemic damage leads to impaired innervation in the infarct region but also in the viable border zone (4). A detailed understanding of the pathophysiology of poorly innervated but viable myocardium is important, because it may precede arrhythmia or contractile failure and hence may be useful for guidance of preventive therapy (5-7).

A common limitation of prior cardiac innervation studies is the use of a single neuronal imaging agent, which provides only a snapshot of neuronal integrity. Reduced tracer uptake may be due to the absence of nerve terminals or due to impaired function (8). These 2 conditions may not be equal with regards to disease progression or regeneration.

PET with multiple catecholamine tracers may provide mechanistic insights into the state of sympathetic nerve terminals (9). If uptake of one catecholamine analog is reduced but another one is avidly retained, functional impairment of a molecular mechanism, which defines the kinetics of the first but not the second agent, is likely, and complete denervation is unlikely. ${ }^{11} \mathrm{C}$-epinephrine, for example, is a physiologic neurotransmitter that is sensitive to degradation by cytosolic monoaminooxidase (10). Retention may be diminished if it is not taken up by nerve terminals or if it is taken up but not stored efficiently in neuronal vesicles (in which it is protected from monoaminooxidase). Combination with ${ }^{11} \mathrm{C}$-hydroxyephedrine, a monoaminooxidase-resistant catecholamine analog (11), may distinguish between impaired catecholamine uptake in general and functional impairment of vesicular storage. Additional combination with ${ }^{11} \mathrm{C}$-phenylephrine, a monoaminooxidase-sensitive catecholamine analog that is inefficiently stored in vesicles and shows degradation even under normal physiologic conditions (12), may be used to confirm the presence or absence of the ability to protect neurotransmitter from metabolic degradation.

Hence, it was our goal to obtain more detailed insights into the molecular alterations underlying impaired innervation in the normally perfused infarct border zone. We studied a porcine model of regional myocardial infarction, in which we previously confirmed the feasibility of PET for detecting perfusion/innervation mismatch and its association with ventricular arrhythmia (13). We speculated that PET with the 3 described radiolabeled catecholamines, integrated with MR imaging and ex vivo tissue analysis, 
would provide mechanistic insights beyond those that are currently available from single neuronal tracer studies.

\section{MATERIALS AND METHODS}

\section{Animal Model and Study Protocol}

The experimental protocol was approved by the Johns Hopkins Institutional Animal Care and Use Committee. Animals were maintained in accordance with the guiding principles of the American Physiologic Society.

Myocardial infarction was induced in 13 young farm pigs $(25-35 \mathrm{~kg}$ ), as previously described $(13,14)$. Under general anesthesia, balloon occlusion of the mid left anterior descending coronary artery, immediately distal to the second diagonal branch, was performed for $150 \mathrm{~min}$. Postoperative treatment included narcotics and nonsteroidal antiinflammatory drugs.

After 4-6 wk, animals underwent 2 imaging sessions within 2-3 d: a basic perfusion/innervation PET session was performed in all animals. This first PET session was followed by a second PET session with 2 additional catecholamine analogs $(n=7)$ or by MR imaging $(n=6)$. After the second session, invasive electrophysiology was performed. Immediately thereafter, animals were sacrificed and hearts were excised for postmortem analysis. Eleven animals completed the protocol. The remaining 2 animals completed noninvasive imaging but died either before or during electrophysiology.

\section{PET}

PET was performed under general anesthesia using an Advance or a Discovery Rx camera (GE Healthcare). After animals were positioned supine, a transmission scan was acquired using external sources or CT, for correction of photon attenuation.

First Session. To measure myocardial perfusion at rest, $400-600 \mathrm{MBq}$ of ${ }^{13} \mathrm{~N}$-ammonia were injected, and dynamic imaging (21 frames; $12 \times 10,6 \times 30$, and $3 \times 300 \mathrm{~s}$ ) was acquired for $20 \mathrm{~min}$. After a break of $40 \mathrm{~min}$ to allow for radioactivity decay, $600-800 \mathrm{MBq}$ of ${ }^{11} \mathrm{C}$ epinephrine, synthesized with a specific activity of more than 37 $\mathrm{GBq}(1 \mathrm{Ci}) / \mathrm{mmol}$ as previously described (15), were injected. Dynamic imaging (14 frames; $6 \times 30,2 \times 60,2 \times 150,2 \times 300$, and $2 \times 600 \mathrm{~s}$ ) was acquired over $40 \mathrm{~min}$.

Second Session. After 2-3 d to allow for recovery, 7 animals were repositioned in the scanner using external skin markers and the laser system of the gantry. ${ }^{11} \mathrm{C}$-phenylephrine and ${ }^{11} \mathrm{C}$-hydroxyephedrine were synthesized at a specific activity of more than $37 \mathrm{GBq}(1 \mathrm{Ci}) / \mathrm{mmol}$ as previously described $(16,17) .{ }^{11} \mathrm{C}$-phenylephrine $(600-800 \mathrm{MBq})$ was injected first, followed by dynamic imaging over $40 \mathrm{~min}$ (14 frames; $6 \times 30,2 \times 60,2 \times 150,2 \times 300$, and $2 \times 600 \mathrm{~s}$ ). After a break of $60 \mathrm{~min}$, 600-800 MBq of ${ }^{11} \mathrm{C}$-hydroxyephedrine were injected, followed by dynamic imaging of $40 \mathrm{~min}$ (14 frames; $6 \times 30,2 \times 60,2 \times 150$, $2 \times 300$, and $2 \times 600 \mathrm{~s}$ ).

For the second PET scan in each session, residual activity from the first injection was ruled out by checking the first frame of the dynamic series, which was acquired before the second dose was injected. The sequence of phenylephrine and hydroxyephedrine scans in session 2 was not randomized because of overall lower myocardial retention and physiologic myocardial washout of phenylephrine, reducing the likelihood of residual activity.

To determine contribution of ${ }^{11} \mathrm{C}$-labeled metabolites to blood activity, venous blood samples were drawn at 1, 5, 10, 20, and $40 \mathrm{~min}$ after injection of each catecholamine, and plasma metabolites were assayed using Sep-Pak cartridges (18).

Quantitative PET Data Processing. Attenuation-corrected transaxial PET images were reconstructed by filtered backprojection. PET images were analyzed by an observer masked to MR imaging and electrophysiology. Using volumetric sampling of the last frame of the perfusion study, we defined myocardial radioactivity in 460 left ventricular (LV) sectors and depicted the radioactivity in a polar map (19). For reproducible and comparable quantitative analysis, the so-defined cardiac long-axis and myocardial segments were automatically transferred to dynamic series of all other tracers, and time-activity curves were obtained, as previously established (20,21). Additionally, for each tracer, arterial input function was defined by a small region of interest in the LV cavity. Absolute myocardial blood flow at rest was quantified using a validated 3-compartment model for ${ }^{13} \mathrm{~N}$-ammonia (17). For the ${ }^{11} \mathrm{C}$-labeled tracers, as previously described $(13,18)$, a retention index was calculated by normalizing myocardial activity at $40 \mathrm{~min}$ to the integral under the metabolite-corrected arterial input function. Finally, to determine clearance, monoexponential fitting of myocardial curves between 5 and 40 min yielded washout rate constants (22).

Polar Map Analysis. To define defect size in percentage of LV myocardium, a threshold of $60 \%$ of the maximum was used (23). The reproducibility of this approach has been previously reported (19). Also, areas of the polar map that showed a matched perfusion/ epinephrine defect (infarct), an epinephrine defect but no perfusion defect (mismatch, border zone), and no defect at all (remote myocardium) were compared.

\section{MR Imaging}

Image Acquisition. MR imaging was performed in 6 animals, using a 3-T human scanner (Achieva; Philips) equipped with vector electrocardiogram technology and a 6-element phased-array cardiac coil. Spiral complementary spatial modulation of magnetization gridtagged images (24) was acquired for motion vector analysis throughout the cardiac cycle. To avoid off-resonance effects due to fat, a spectral spatial excitation was used. Additionally, cine imaging and delayedenhancement imaging of gadolinium-diethylenetriaminepentaacetic acid was performed.

Data Analysis. MR imaging was analyzed by an interpreter masked to PET results. In cine and delayed-enhancement images, endocardium and epicardium were manually contoured at each short-axis level in end systole and end diastole to calculate LV volumes, ejection fraction, mass, and regional wall thickening and thickness. Harmonic phase analysis was conducted for tagged studies to determine peak circumferential myocardial strain throughout the cardiac cycle and time to peak strain in 8 segments of LV myocardium (25). For accurate matching with PET, septum on all MR imaging studies was defined as the area between anterior and posterior insertion of the right ventricle, and the other myocardial walls were defined accordingly. The apex, which is not appropriately imaged by short-axis images, was excluded.

\section{Electrophysiologic Testing}

An abbreviated invasive electrophysiologic workup consisted of programmed stimulation to identify inducibility of ventricular tachycardia (VT) as previously described in more detail (13).

\section{Postmortem Analysis}

After completion of the study protocol, anesthetized pigs were euthanized ( $4 \mathrm{mmol} / \mathrm{L}$ potassium chloride intravenously). Hearts were immediately removed, and gross macroscopic LV short-axis slices were created. Under guidance by PET images and gross morphology, tissue samples were collected from the central infarct region, the infarct border zone at the location of PET perfusion/innervation mismatch, and the remote noninfarct region, for further analysis. Samples were frozen and sliced for general histology by hematoxylin and eosin staining. Additionally, immunohistochemical staining was performed using anti-tyrosine hydroxylase $(\mathrm{TH})$ antibody to detect sympathetic nerve fibers and anti-growth-associated protein 43 (GAP43) antibody to detect nerve sprouts, as previously described (monoclonal mouse anti-GAP43 and anti-TH, respectively, 1:50 
dilution; Chemicon International, Inc.) (26). Image J software (National Institutes of Health) was used to quantify the percentage of intact tissue in hematoxylin and eosin stains and to determine the number of positively stained nerve fibers per field of view in TH and GAP43 stains (26).

\section{Statistical Analysis}

Statistical analysis was performed using Medcalc software. All data are shown as mean $\pm \mathrm{SD}$. A $P$ value of less than 0.05 was considered statistically significant. The Mann-Whitney $U$ test was used to compare groups with and without VT inducibility. The Wilcoxon test was used to compare global perfusion and innervation defect sizes and mismatch and remote myocardium intraindividually. The KruskalWallis test was used for comparison of PET and MR imaging variables between 3 types of segments, and the Friedman test was used to compare defects from 3 different catecholamine tracers. Pearson correlation was used to describe a univariate relationship between continuous variables. Multivariate stepwise regression analysis was performed to identify independent determinants of MR imagingderived strain parameters. To support the assumption of the independence of myocardial segments, a correlation analysis was performed using the most important PET variable, the mismatch between perfusion and innervation abnormality. Values in the distal anterior wall (the predominant site of mismatch) did not correlate with corresponding values from any of the 8 other segments.

\section{RESULTS}

\section{Innervation/Perfusion Mismatch in Infarct Border Zone}

In all animals, the global perfusion defect size was $35 \% \pm 12 \%$ $\mathrm{LV}$, and the epinephrine retention defect size was significantly larger at $42 \% \pm 12 \%(P<0.001)$. This difference in defect sizes resulted in an innervation/perfusion mismatch comprising $7 \% \pm$ $4 \%$ of the left ventricle in the infarct border zone (Fig. 1). Pro- grammed stimulation showed VT inducibility in 5 of 11 animals. Consistent with prior work (13), animals with inducible VT had significantly larger innervation/perfusion mismatch $(11 \pm 4$ vs. $5 \% \pm 3 \% \mathrm{LV} ; P=0.03)$.

\section{Innervation/Perfusion Mismatch and Regional Strain}

In the MR imaging subgroup $(n=6), \mathrm{LV}$ ejection fraction was $35 \% \pm 9 \%$ and there was mostly anteroseptal transmural delayed enhancement. Six of 48 myocardial segments showed a matched reduction of perfusion and relative epinephrine retention, and 10 showed innervation/perfusion mismatch. In mismatch segments, perfusion, absolute flow, and end-diastolic wall thickness were similar to normal segments, whereas epinephrine retention, wall thickening, and peak strain were significantly impaired when compared with normal segments (Table 1; Fig. 2). Peak strain correlated significantly with regional perfusion $(\mathrm{r}=-0.55 ; P<0.001)$ and epinephrine retention $(\mathrm{r}=-0.64 ; P<0.001)$, but only epinephrine retention was an independent correlate at stepwise multiple regression analysis.

\section{Epinephrine, Hydroxyephedrine, and Phenylephrine Handling}

In the dual-PET-session subgroup $(n=7)$, defect size for the physiologic neurotransmitter epinephrine was larger than the perfusion defect (Fig. 3). Hydroxyephedrine defect, however, was significantly smaller than epinephrine and not different from perfusion defect size. Phenylephrine defect was similar to epinephrine but larger than hydroxyephedrine and perfusion defect.

Table 2 summarizes retention and washout kinetics of the 3 tracers in myocardial regions. Reduced regional retention in the viable infarct border zone was due to significantly elevated washout of epinephrine and phenylephrine, whereas there was no washout of hydroxyephedrine.

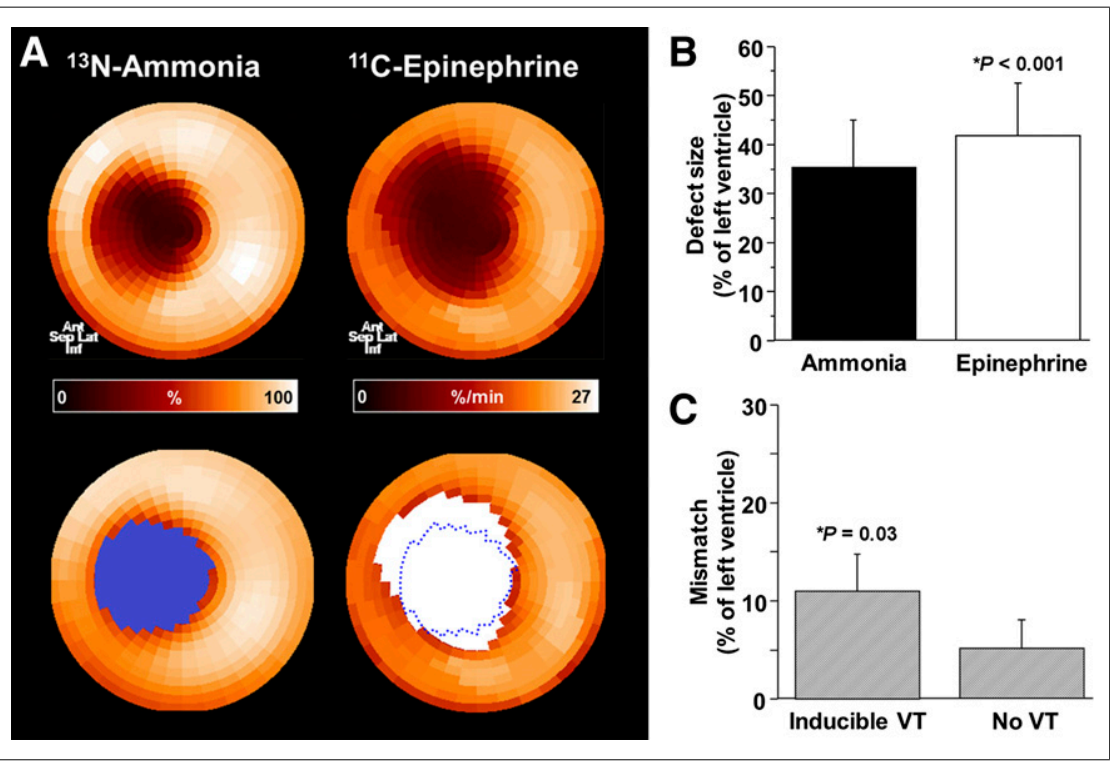

FIGURE 1. Perfusion/innervation mismatch after myocardial infarction. (A) Representative PET polar maps of myocardial perfusion $\left({ }^{13} \mathrm{~N}\right.$-ammonia) and innervation $\left({ }^{11} \mathrm{C}\right.$-epinephrine). Innervation defect (white area) is larger than perfusion defect (blue area), resulting in normally perfused myocardium with impaired innervation in infarct border zone. (B) Bar chart showing defect sizes in all animals $(n=13)$. (C) Bar chart comparing perfusion/innervation mismatch in subgroups with $(n=5)$ and without $(n=6)$ inducible VT. Ant $=$ anterior; inf $=$ inferior; lat $=$ lateral; sep $=$ septal.

\section{Ex Vivo Analysis}

Histology confirmed extensive tissue damage in the infarct and mild damage but mostly preserved tissue integrity in the border zone (Fig. 4). Immunostaining in the infarct area was not conclusive because of the lack of viable tissue. In the border zone, immunohistochemistry revealed the presence of TH-positive nerve fibers and GAP43positive nerve sprouts. Quantitatively, the number of TH- $(0.58 \pm 0.14$ vs. $0.79 \pm 0.24$ per field of view; $P=0.28)$ and GAP43positive fibers $(0.83 \pm 0.29$ vs. $1.52 \pm$ 0.56 per field of view; $P=0.08$ ) was not different from remote myocardium.

\section{DISCUSSION}

Our study confirms prior experimental and clinical work, which showed impaired sympathetic innervation in the viable infarct border zone that has electrophysiologic implications and contributes to ventricular arrhythmia $(3,7,13)$. Integrated analysis of PET and MR imaging also shows that an innervation/perfusion mismatch after myocardial infarction is associated with altered regional contractility and 
TABLE 1

PET and MR Imaging Results in Myocardial Segments

\begin{tabular}{|c|c|c|c|}
\hline Segmental pattern & Normal $(n=32)$ & Mismatch $(n=10)$ & Scar $(n=6)$ \\
\hline Relative perfusion (\% of LV maximum) & $82 \pm 9$ & $78 \pm 7$ & $40 \pm 7^{\star \star 十}$ \\
\hline Absolute flow (mL/min/g) & $0.76 \pm 0.21$ & $0.79 \pm 0.21$ & $0.49 \pm 0.19^{\star \dagger}$ \\
\hline Relative epinephrine uptake (\% of LV maximum) & $84 \pm 9$ & $66 \pm 10^{*}$ & $39 \pm 6^{\star \dagger}$ \\
\hline Epinephrine retention index (\%/min) & $19.5 \pm 3.4$ & $17.3 \pm 2.0^{\star}$ & $9.6 \pm 1.3^{\star \dagger}$ \\
\hline End-diastolic wall thickness (mm) & $7 \pm 1$ & $7 \pm 1$ & $5 \pm 1^{\star+}$ \\
\hline Wall thickening (\%) & $46 \pm 28$ & $24 \pm 25^{\star}$ & $8 \pm 12^{*}$ \\
\hline Peak strain (\%) & $-14 \pm 4$ & $-8 \pm 5^{*}$ & $-4 \pm 5^{\star}$ \\
\hline Time to peak strain (\% of RR interval) & $50 \pm 7$ & $52 \pm 8$ & $70 \pm 7^{\star \dagger}$ \\
\hline $\begin{array}{l}{ }^{*} P<0.05 \text { vs. normal. } \\
{ }^{\dagger} P<0.05 \text { vs. mismatch. } \\
\text { RR }=\text { cardiac cycle. }\end{array}$ & & & \\
\hline
\end{tabular}

wall strain despite preserved perfusion and viability. Most importantly, however, interrogation of the myocardial kinetics of multiple different catecholamine analogs suggests that the innervation/ perfusion mismatch in the infarct border zone is associated with functionally impaired innervation in our model. Neuronal vesicular catecholamine storage and protection from metabolic degradation are more severely altered than catecholamine uptake.

In healthy myocardium, the physiologic catecholamine epinephrine is taken up by sympathetic nerve terminals through the norepinephrine transporter and then stored in neuronal vesicles, where it is protected from monoaminooxidase degradation $(10,18)$.

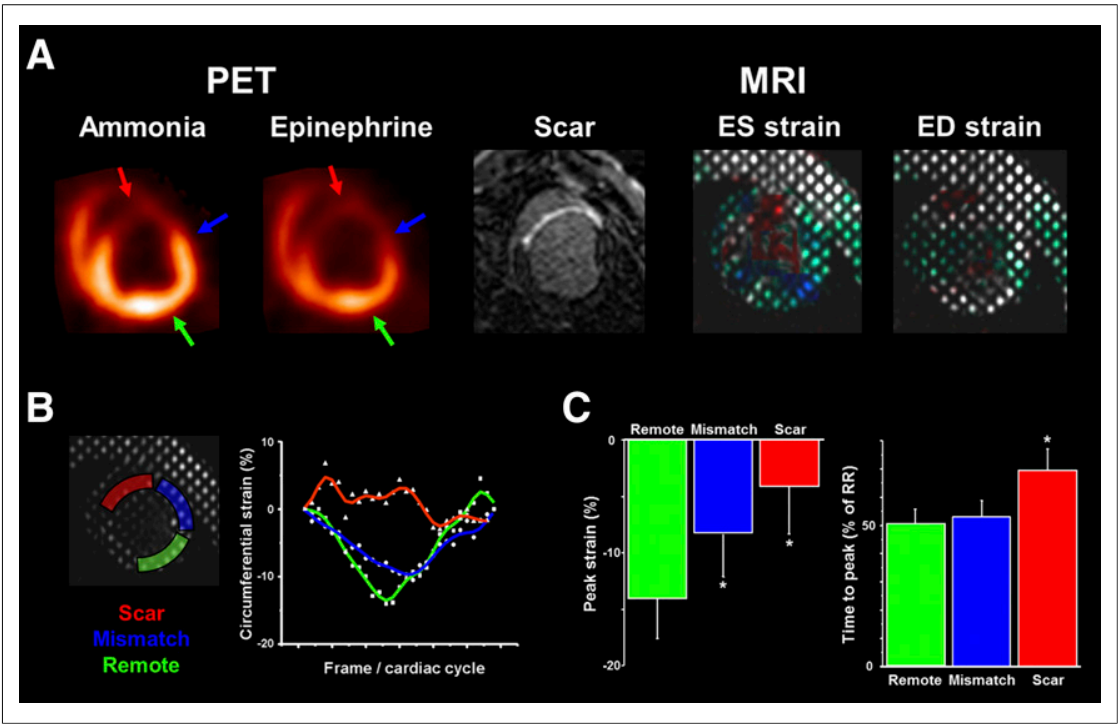

FIGURE 2. Circumferential myocardial strain analysis. (A) Matched short-axis slices of mid/ distal left ventricle in representative animal. PET perfusion (using ${ }^{13} \mathrm{~N}$-ammonia) and MR imaging delayed enhancement (scar) show anteroseptal infarct. PET innervation (using ${ }^{11} \mathrm{C}$-epinephrine) shows defect, which exceeds perfusion defect (innervation/perfusion mismatch). Color-coded tagged MR imaging of circumferential strain is shown on right. (B) Regional strain in representative segments. Curves throughout cardiac cycle are shown for scar (red), mismatch (blue), and normally perfused remote myocardium (green). Infarct curve is irregular with attenuated and delayed nadir. Nadir in mismatch region is attenuated versus remote. (C) Bar charts comparing peak strain and time to peak in 3 different groups of segments $\left({ }^{*} P<0.05\right.$ vs. remote). $E D=$ end diastolic; ES = end systolic.
In our study, epinephrine retention is reduced in the normally perfused infarct border zone. In contrast, hydroxyephedrine, a catecholamine analog that is an avid substrate for neuronal transport but is resistant to monoaminooxidase degradation (11) and therefore depends less on vesicular storage for neuronal retention, showed a smaller defect than epinephrine. This smaller defect suggests that neuronal uptake mechanisms may at least be partially preserved in the infarct border zone, whereas vesicular storage is more severely impaired. The presence of increased washout of epinephrine in the border zone provides further support, as do the findings using a third catecholamine, phenylephrine. This monoaminooxidasesensitive neurotransmitter analog is taken up by nerve terminal and stored in vesicles, but even under physiologic conditions there is vesicular leakage and subsequent washout $(12,22)$. Concordant with epinephrine, regional phenylephrine retention was reduced in the infarct border zone and washout was increased, confirming the presence of impaired vesicular storage rather than the complete absence of intact nerve terminals in this region in our model of well-defined, regional myocardial damage.

Immunohistologic detection of intact nerve fibers and nerve sprouting in the infarct border zone of our model is consistent with prior work $(27,28)$ and provides more support of a functional impairment of sympathetic innervation. Of note, the histologic presence of nerve fibers alone does not allow for conclusions about their functional state. Molecular imaging provides further biologic insights and suggests that neuronal transport may still be intact in histologically detectable nerves, whereas vesicular storage is substantially impaired.

Some of our findings may be specific to our animal model. First, myocardial infarction was induced in otherwise healthy, 


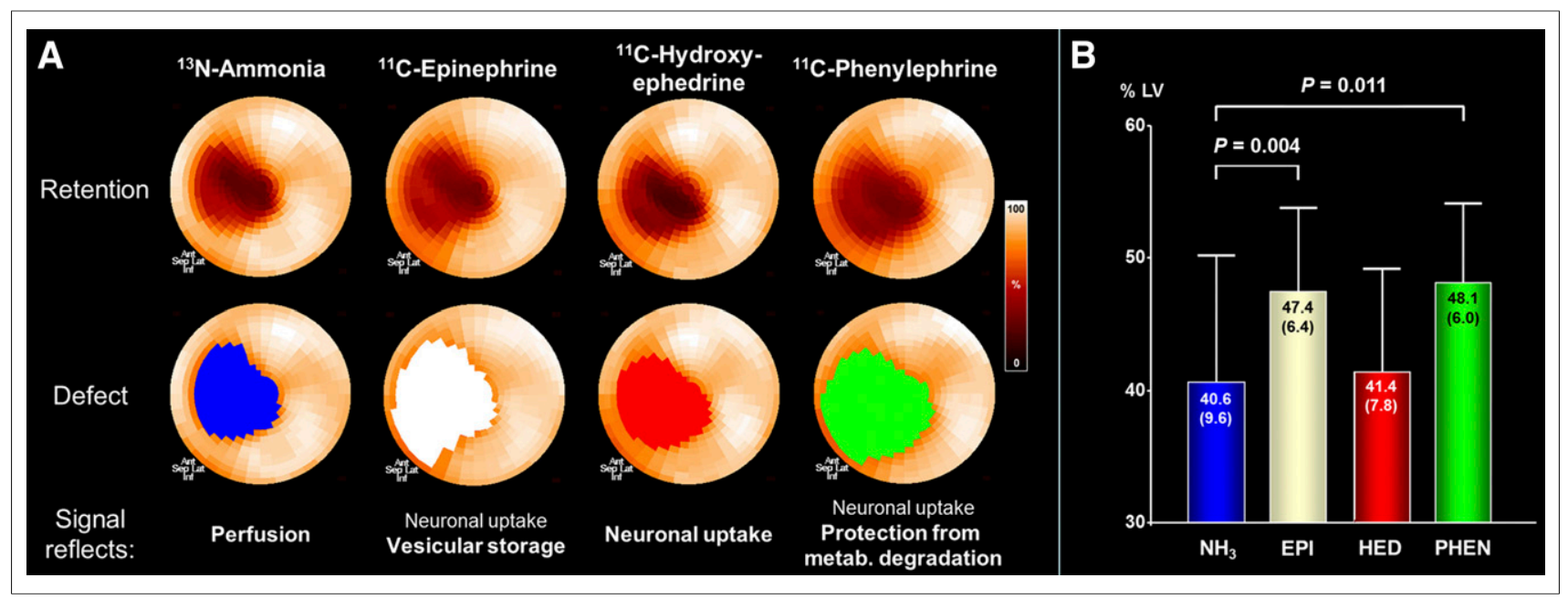

FIGURE 3. Characterization of myocardial innervation using multiple radiolabeled catecholamines. (A) Polar maps of representative infarct animal showing retention of 4 tracers (top), along with color-coded defect areas. Epinephrine and phenylephrine defects exceed perfusion defect, whereas hydroxyephedrine defect is comparable to perfusion. (B) Bar chart showing group results of defect sizes. Ant $=$ anterior; $E P I=e p i n e p h r i n e ; ~ H E D=$ hydroxyephedrine; inf = inferior; lat = lateral; metab. = metabolic; $\mathrm{NH}_{3}=$ ammonia; $\mathrm{PHEN}=$ phenylephrine; sep = septal.

young pigs without concomitant disease. In the clinical situation, infarction is associated with more or less extensive coronary artery disease. There, repetitive ischemic insults may result in more severe neuronal damage in otherwise viable myocardium and, hence, in more severe defects for the uptake marker hydroxyephedrine. Consistently, a prior study using hydroxyephedrine in humans showed retention defects exceeding the perfusion defect in a fraction, but not all, of patients early after myocardial infarction (29). Also, the recent PAREPET study, which identified hydroxyephedrine defects exceeding perfusion defects and predicting arrhythmia risk (30), was conducted in subjects with ischemic cardiomyopathy and heart failure and thus covered a more severe state of ischemic damage. Our animal model does not reflect chronic ischemia or multivessel disease or longstanding heart failure. The present results thus probably reflect a less profound state of neuronal impairment, which may precede a more severe impairment in advanced ischemic heart disease.
The primary purpose of our work was to highlight the fact that impaired sympathetic innervation is not a black-and-white phenomenon of denervation or innervation, but that there are transitional stages of incomplete molecular impairment that can be identified using multiple catecholamine imaging agents. The proof of feasibility for this approach is provided in the present study. The practical implications of molecularly impaired but not completely denervated myocardium for the clinical course of ischemic heart disease will need to be clarified in subsequent work. Our initial results may serve as a basis for this.

Second, species differences in catecholamine kinetics have been reported. In pigs, extraneuronal clearance of catecholamines by the uptake-2 system is more pronounced than in primates (31). Also, there may be differences in monoaminooxidase activity (32), which may interfere with intraneuronal washout rates. But we combined 3 tracers to determine nerve terminal function, and relative comparison of their kinetics can be done independent of species

TABLE 2

Kinetics of 3 Radiolabeled Catecholamines After Myocardial Infarction

\begin{tabular}{|c|c|c|c|}
\hline Myocardial region & Normal & Mismatch & Scar \\
\hline \multicolumn{4}{|l|}{ Epinephrine } \\
\hline Retention index (\%/min) & $27.2 \pm 5.4$ & $18.1 \pm 3.1^{*}$ & $9.9 \pm 1.3^{\star} \dagger$ \\
\hline Washout rate (\%/min) & $1.1 \pm 0.3$ & $1.5 \pm 0.4^{\star}$ & $\mathrm{NA}^{\ddagger}$ \\
\hline \multicolumn{4}{|l|}{ Hydroxyephedrine } \\
\hline Retention index (\%/min) & $17.3 \pm 6.3$ & $13.5 \pm 4.7$ & $6.2 \pm 2.5^{\star} \dagger$ \\
\hline Washout rate (\%/min) & $\complement^{\S}$ & $\square^{\S}$ & $\mathrm{NA}^{\ddagger}$ \\
\hline \multicolumn{4}{|l|}{ Phenylephrine } \\
\hline Retention index (\%/min) & $16.1 \pm 2.6$ & $11.5 \pm 1.5^{\star}$ & $5.9 \pm 0.6^{\star \dagger}$ \\
\hline Washout rate (\%/min) & $0.8 \pm 0.3$ & $1.2 \pm 0.3^{*}$ & $\mathrm{NA}^{\ddagger}$ \\
\hline \multicolumn{4}{|l|}{$\begin{array}{l}{ }^{\star} P<0.05 \text { vs. normal. } \\
{ }^{\dagger} P<0.05 \text { vs. mismatch. }\end{array}$} \\
\hline
\end{tabular}




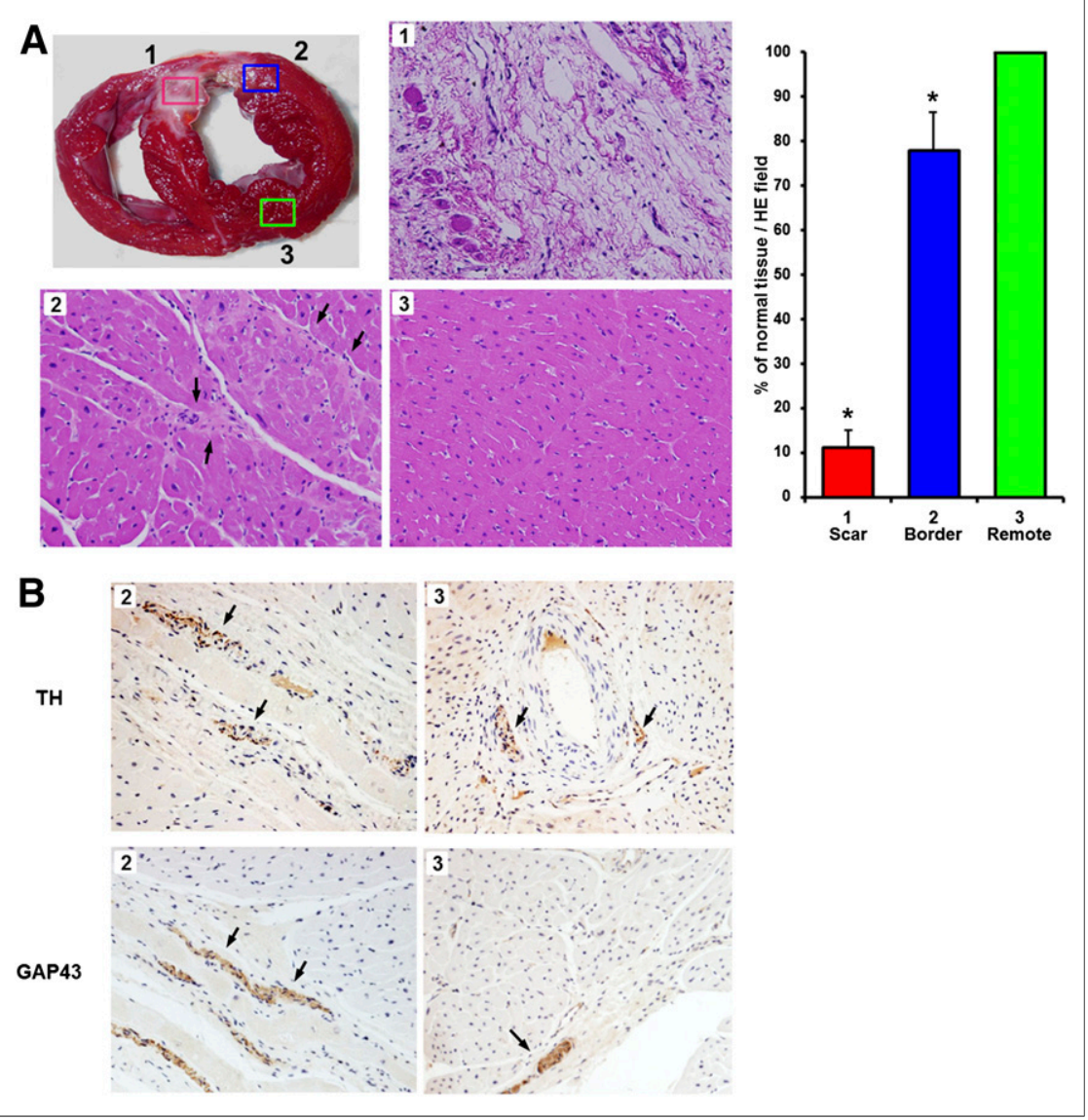

FIGURE 4. Ex vivo tissue analysis. (A) Gross morphologic short-axis slice (top left) indicates location of sampling for microscopic analysis from scar (1), border zone (2), and remote area (3). Hematoxylin/eosin (HE) stains show extensive tissue damage in scar, mild damage/fibrosis in border zone (arrows), and preserved integrity in remote area. Quantitative analysis (bar chart, right) confirms visual impression ( ${ }^{*} P<0.01$ vs. remote region). (B) Immunohistochemical stains for tyrosine hydroxylase and GAP43 show brown-stained nerve fibers/sprouting nerves (arrows) in infarct border zone and remote region.

properties. Third, the dependency of hydroxyephedrine and epinephrine on vesicular storage requires some additional discussion. It has been reported that reserpine, a blocker of vesicular storage, reduces hydroxyephedrine retention in vivo by up to $50 \%$ (33), but this may be partially attributed to systemic effects (resulting from competition by increased norepinephrine levels and effects on ventricular loading). Other studies in isolated perfused hearts have shown a more severe, almost complete blockage of epinephrine retention by reserpine (10). Taken together with the effect of a desipramine chase on hydroxyephedrine only (9) and the monoaminooxidase sensitivity of epinephrine, necessitating vesicular storage for protection from metabolism, epinephrine retention is clearly more dependent on vesicular storage than that of hydroxyephedrine. Fourth, all scans were obtained during isoflurane anesthesia. Isoflurane increases sympathetic tone (34), but the net effect on kinetics of all 3 tracers is expected to be similar so that relative comparison of their kinetics is not affected. And finally, spatial correlation of histology with imaging is challenging. We used the infarct region as a landmark, but misalignment cannot be completely excluded. It should be considered, however, that our histologic analysis confirms prior work, which provides some validation to the approach.

Our work may have practical implications: Speculatively, although not proven in this study, recovery of a state of impaired nerve function may be easier to achieve than in the case of complete denervation. Also, whereas the current clinical focus for innervation imaging is on heart failure (30, 35), studies such as ours suggest that additional focus on acute myocardial infarction as a precursor of arrhythmia and heart failure, and on the analysis of regional abnormalities rather than global impairments, could be valuable. And finally, whereas a more widespread use of the ${ }^{11} \mathrm{C}$ tracers used in this project cannot be expected because of the dependency on a cyclotron and on-site radiopharmacy, our work suggests that the kinetics of more widely available neuronal imaging agents such as ${ }^{123}$ I-metaiodobenzylguanidine, or a new ${ }^{18} \mathrm{~F}$-labeled neuronal PET imaging agent (36), should be carefully evaluated to identify the major molecular mechanisms that determine their kinetics and the respective imaging signal.

\section{CONCLUSION}

Using a complex PET imaging protocol with multiple catecholamines and adjunct functional and electrophysiologic tests in an established pig model of myocardial infarction, the present study suggests that neuronal vesicular catecholamine storage and protection from metabolic degradation are more severely altered than catecholamine uptake in the viable infarct border zone. This alteration may reflect an intermediate state between normal innervation and complete denervation. These results provide valuable pathobiologic insights, and they may serve as a foundation for the design of future studies investigating the clinical value of neuronal imaging in more depth.

\section{DISCLOSURE}

The costs of publication of this article were defrayed in part by the payment of page charges. Therefore, and solely to indicate this fact, this article is hereby marked "advertisement" in accordance with 18 USC section 1734. This study was supported by the Donald W. Reynolds Foundation and the W.W. Smith Charitable Trust. No other potential conflict of interest relevant to this article was reported.

\section{REFERENCES}

1. Bengel FM, Barthel P, Matsunari I, Schmidt G, Schwaiger M. Kinetics of I-123metaiodobenzylguanidine after acute myocardial infarction and reperfusion therapy. J Nucl Med. 1999;40:904-910.

2. McGhie AI, Corbett JR, Akers MS, et al. Regional cardiac adrenergic function using I-123 meta-iodobenzylguanidine tomographic imaging after acute myocardial infarction. Am J Cardiol. 1991;67:236-242.

3. Simões MV, Barthel P, Matsunari I, et al. Presence of sympathetically denervated but viable myocardium and its electrophysiologic correlates after early revascularised, acute myocardial infarction. Eur Heart J. 2004;25:551-557.

4. Matsunari I, Schricke U, Bengel FM, et al. Extent of cardiac sympathetic neuronal damage is determined by the area of ischemia in patients with acute coronary syndromes. Circulation. 2000;101:2579-2585. 
5. Arora R, Ferrick KJ, Nakata T, et al. I-123 MIBG imaging and heart rate variability analysis to predict the need for an implantable cardioverter defibrillator. J Nucl Cardiol. 2003;10:121-131.

6. Kramer CM, Nicol PD, Rogers WJ, et al. Reduced sympathetic innervation underlies adjacent noninfarcted region dysfunction during left ventricular remodeling. J Am Coll Cardiol. 1997;30:1079-1085.

7. Sakata K, Mochizuki M, Yoshida H, et al. Cardiac sympathetic dysfunction contributes to left ventricular remodeling after acute myocardial infarction. Eur J Nucl Med. 2000;27:1641-1649.

8. Bengel FM. Imaging targets of the sympathetic nervous system of the heart: translational considerations. J Nucl Med. 2011;52:1167-1170.

9. Tipre DN, Fox JJ, Holt DP, et al. In vivo PET imaging of cardiac presynaptic sympathoneuronal mechanisms in the rat. J Nucl Med. 2008;49:1189-1195.

10. Nguyen NT, DeGrado TR, Chakraborty P, Wieland DM, Schwaiger M. Myocardial kinetics of carbon-11-epinephrine in the isolated working rat heart. J Nucl Med. 1997;38:780-785.

11. Raffel DM, Chen W, Sherman PS, Gildersleeve DL, Jung YW. Dependence of cardiac ${ }^{11} \mathrm{C}$-meta-hydroxyephedrine retention on norepinephrine transporter density. J Nucl Med. 2006;47:1490-1496.

12. Raffel DM, Wieland DM. Influence of vesicular storage and monoamine oxidase activity on $\left[{ }^{11} \mathrm{C}\right]$ phenylephrine kinetics: studies in isolated rat heart. J Nucl Med. 1999;40:323-330.

13. Sasano T, Abraham MR, Chang KC, et al. Abnormal sympathetic innervation of viable myocardium and the substrate of ventricular tachycardia after myocardial infarction. J Am Coll Cardiol. 2008;51:2266-2275.

14. Sasano T, McDonald AD, Kikuchi K, Donahue JK. Molecular ablation of ventricular tachycardia after myocardial infarction. Nat Med. 2006;12:1256-1258.

15. Chakraborty PK, Gildersleeve DL, Jewett DM, et al. High yield synthesis of high specific activity R-(-)-[ $\left.{ }^{11} \mathrm{C}\right]$ epinephrine for routine PET studies in humans. Nucl Med Biol. 1993;20:939-944.

16. Del Rosario RB, Jung YW, Caraher J, Chakraborty PK, Wieland DM. Synthesis and preliminary evaluation of $\left[{ }^{11} \mathrm{C}\right]-(-)$-phenylephrine as a functional heart neuronal PET agent. Nucl Med Biol. 1996;23:611-616.

17. Schwaiger M, Guibourg H, Rosenspire K, et al. Effect of regional myocardial ischemia on sympathetic nervous system as assessed by fluorine-18-metaraminol. J Nucl Med. 1990;31:1352-1357.

18. Münch G, Nguyen NTB, Nekolla S, et al. Evaluation of sympathetic nerve terminals with $\left[{ }^{11} \mathrm{C}\right]$ epinephrine and $\left[{ }^{11} \mathrm{C}\right]$ hydroxyephedrine and positron emission tomography. Circulation. 2000;101:516-523.

19. Nekolla SG, Miethaner C, Nguyen N, Ziegler SI, Schwaiger M. Reproducibility of polar map generation and assessment of defect severity and extent assessment in myocardial perfusion imaging using positron emission tomography. Eur J Nucl Med. 1998;25:1313-1321.

20. Bengel FM, Permanetter B, Ungerer M, Nekolla SG, Schwaiger M. Relationship between altered sympathetic innervation, oxidative metabolism and contractile function in the cardiomyopathic human heart: a non-invasive study using positron emission tomography. Eur Heart J. 2001;22:1594-1600.
21. Bengel FM, Ueberfuhr P, Schiepel N, Nekolla SG, Reichart B, Schwaiger M. Myocardial efficiency and sympathetic reinnervation after orthotopic heart transplantation: a noninvasive study with positron emission tomography. Circulation. 2001;103:1881-1886.

22. Raffel DM, Corbett JR, del Rosario RB, et al. Sensitivity of $\left[{ }^{11} \mathrm{C}\right]$ phenylephrine kinetics to monoamine oxidase activity in normal human heart. $\mathrm{J} \mathrm{Nucl} \mathrm{Med}$. 1999;40:232-238.

23. Delbeke D, Lorenz CH, Votaw JR, et al. Estimation of left ventricular mass and infarct size from nitrogen-13-ammonia PET images based on pathological examination of explanted human hearts. J Nucl Med. 1993;34:826-833.

24. Ryf S, Kissinger KV, Spiegel MA, et al. Spiral MR myocardial tagging. Magn Reson Med. 2004;51:237-242.

25. Osman NF, Kerwin WS, McVeigh ER, Prince JL. Cardiac motion tracking using CINE harmonic phase (HARP) magnetic resonance imaging. Magn Reson Med. 1999;42:1048-1060.

26. Liu YB, Wu CC, Lu LS, et al. Sympathetic nerve sprouting, electrical remodeling, and increased vulnerability to ventricular fibrillation in hypercholesterolemic rabbits. Circ Res. 2003;92:1145-1152.

27. Cao JM, Fishbein MC, Han JB, et al. Relationship between regional cardiac hyperinnervation and ventricular arrhythmia. Circulation. 2000;101:1960-1969.

28. Chen P-S, Chen LS, Cao J-M, Sharifi B, Karagueuzian HS, Fishbein MC. Sympathetic nerve sprouting, electrical remodeling and the mechanisms of sudden cardiac death. Cardiovasc Res. 2001;50:409-416.

29. Allman KC, Wieland DM, Muzik O, Degrado TR, Wolfe ER Jr, Schwaiger M. Carbon-11 hydroxyephedrine with positron emission tomography for serial assessment of cardiac adrenergic neuronal function after acute myocardial infarction in humans. J Am Coll Cardiol. 1993;22:368-375.

30. Fallavollita JA, Heavey BM, Luisi AJ Jr, et al. Regional myocardial sympathetic denervation predicts the risk of sudden cardiac arrest in ischemic cardiomyopathy. J Am Coll Cardiol. 2014;63:141-149.

31. Carr EA Jr, Carroll M, Counsell RE, Tyson JW. Studies of uptake of the bretylium analogue, iodobenzyltrimethylammonium iodide, by non-primate, monkey and human hearts. Br J Clin Pharmacol. 1979;8:425-432.

32. Callingham BA. Comparative aspects of monoamine oxidase. Vet Res Commun. 1983;7:325-330.

33. Nomura Y, Matsunari I, Takamatsu H, et al. Quantitation of cardiac sympathetic innervation in rabbits using ${ }^{11} \mathrm{C}$-hydroxyephedrine PET: relation to ${ }^{123} \mathrm{I}-\mathrm{MIBG}$ uptake. Eur J Nucl Med Mol Imaging. 2006;33:871-878.

34. Tanaka S, Tsuchida H, Namba H, Namiki A. Clonidine and lidocaine inhibition of isoflurane-induced tachycardia in humans. Anesthesiology. 1994;81:13411349.

35. Jacobson AF, Senior R, Cerqueira MD, et al. Myocardial iodine-123 metaiodobenzylguanidine imaging and cardiac events in heart failure: results of the prospective ADMIRE-HF (AdreView Myocardial Imaging for Risk Evaluation in Heart Failure) study. J Am Coll Cardiol. 2010;55:2212-2221.

36. Yu M, Bozek J, Lamoy M, et al. Evaluation of LMI1195, a novel ${ }^{18} \mathrm{~F}$-labeled cardiac neuronal PET imaging agent, in cells and animal models. Circ Cardiovasc Imaging. 2011;4:435-443. 